\title{
tic\&société
}

Vol. 9, No 1-2 | 1 er semestre 2015 - 2ème semestre 2015

Les religions au temps du numérique

\section{Montrer « l'exorcisme » de Sarah Palin sur le web.}

Enquête(s) sur la portée politique d'images religieuses

\section{Philippe GONZALEZ}

\section{(2) OpenEdition \\ Journals}

\section{Electronic version}

URL: http://journals.openedition.org/ticetsociete/1826

DOI: 10.4000/ticetsociete. 1826

\section{Publisher}

Association ARTIC

\section{Electronic reference}

Philippe GONZALEZ, « Montrer « l'exorcisme » de Sarah Palin sur le web. », tic\&société [Online], Vol. 9, N

- 1-2 | 1er semestre 2015 - 2ème semestre 2015, Online since 08 January 2016, connection on 19

April 2019. URL : http://journals.openedition.org/ticetsociete/1826 ; DOI : 10.4000/ticetsociete.1826 
tic\&société - 9 (1-2), 2015

Montrer « l'exorcisme » de Sarah Palin sur le web.

Enquête(s) sur la portée politique d'images

religieuses

Philippe GONZALEZ

Institut des sciences sociales

Faculté des sciences sociales et politiques

Université de Lausanne

Bâtiment Géopolis, bureau 5122, 1015 Lausanne, Suisse

philippe.gonzalez@unil.ch 


\section{Montrer "l'exorcisme » de Sarah Palin sur le web. Enquête(s) sur la portée politique d'images religieuses}

Philippe GONZALEZ est maître d'enseignement et de recherche en sociologie à l'Université de Lausanne, et membre associé du Centre d'étude des mouvements sociaux (EHESS). Son travail porte sur le statut des religions dans l'espace public, à l'articulation des enjeux politiques. En 2014, il a publié Que ton règne vienne : des évangéliques tentés par le pouvoir absolu (Genève, Labor et Fides). II a notamment dirigé Le religieux entre science et cité (avec Christophe Monnot, Genève, Labor et Fides, 2012) et Quel âge post-séculier? Religions, démocraties, sciences (avec Joan Stavo-Debauge et Roberto Frega, Paris, Editions de l'EHESS, 2015).

Résumé : En septembre 2008, une vidéo est diffusée sur internet montrant Sarah Palin, alors candidate à la viceprésidence des États-Unis, en train de recevoir " une protection contre la sorcellerie » lors d'une célébration religieuse. La séquence est aussitôt reprise par les médias du monde entier qui s'interrogent et enquêtent sur les rapports entre ces images et l'engagement politique de la républicaine. Cet article suit la circulation de la vidéo au travers de différentes scènes de visibilité, allant des médias généralistes au site internet de l'Église où elle a été tournée, en passant par un blog qui a attiré l'attention publique sur ces images. L'enquête met au jour comment ce film a été compris dans ces différents contextes, et montre les effets de ce travail d'interprétation en 2011, suite à l'avènement du Tea Party et à l'horizon de la prochaine échéance présidentielle. La vidéo qui, en 2008, apparaissait dans les médias généralistes comme une illustration de la «bigoterie » de Palin a contribué à figurer, en 2011, une menace « théocratique » pour la démocratie américaine.

Mots clés: Droite chrétienne, espace public, monstration, religion et politique, socio-sémiotique.

Abstract: Showing Sarah Palin's "Exorcism" on the Web. Inquiries into the Political Impact of Religious Images - In September 2008, a video appeared on the Internet showing Sarah Palin, then vice-presidential candidate, receiving "a protection against witchcraft" during a religious celebration. The 
sequence was immediately taken up by the international media, who asked questions and investigated the connections between these images and the political activities of the Republican. This article follows the circulation of the video through different scenes of visibility, going from the mainstream media to the Internet website of the church where it was shot, by way of a blog which drew public attention to these images. The inquiry brings to light how this film was understood in various contexts, and shows the impact of this interpretative work in 2011, with the advent of the Tea Party and within the horizon of the next presidential election. In 2008, the video appeared in the mainstream media as an illustration of the Palin's "bigotry"; in 2011 , it helped delineate a "theocratic" threat posed to American democracy.

Keywords: Christian Right, public sphere, monstration, religion and politics, social semiotics.

Resumen : Mostrar el " exorcismo » de Sarah Palin en la web. Investigaciones sobre el significado político de las imágenes religiosas - En septiembre de 2008, un video se transmite en Internet mostrando a Sarah Palin, entonces candidata a la vicepresidencia de Estados Unidos, recibiendo una "protección contra la brujería" a través de una celebración religiosa. La secuencia fue recogida inmediatamente por los medios de comunicación de todo el mundo, que investigan las conexiones entre estas imágenes y las actividades políticas de la republicana. Este artículo hace un seguimiento de la circulación del video a través de diferentes escenas de visibilidad, que van desde los principales medios de comunicación a la página Internet de la Iglesia en donde se rodó, pasando por un blog que llamó la atención del público sobre estas imágenes. La investigación pone de manifiesto cómo fue interpretado este video en varios contextos, y muestra el impacto de este trabajo interpretativo en 2011, tras el lanzamiento del Tea Party, en el horizonte de las próximas elecciones presidenciales. En 2008, el video apareció en los principales medios de comunicación como una ilustración del "fanatismo" de Palin; en 2011, se configuró como una amenaza "teocrática" contra la democracia estadounidense.

Palabras clave: Derecha Cristiana, esfera pública, mostración, religión y política, socio-semiótica. 
«Sarah Palin reçoit une protection contre les sorcières » - c'est le titre d'une vidéo postée sur Youtube, en septembre 2008, par un petit média indépendant, Irregular Times ${ }^{1}$. On y voit la candidate à la vice-présidence étasunienne se faire imposer les mains, dans le cadre d'une cérémonie religieuse, afin d'être protégée contre «toute forme de sorcellerie ${ }^{2}$. En deux jours, ces images font le tour du monde. Les médias s'interrogent sur la signification de cette « bénédiction » pour l'actualité politique étasunienne. Comment penser ensemble des activités aussi disparates au sein d'une démocratie libérale: la fonction politique de la candidate et «l'exorcisme »? Le mystère qui entoure cette vidéo est d'autant plus grand que Sarah Palin est une relative inconnue sur la scène politique étasunienne.

Cet article s'intéresse à la façon dont différents acteurs sociaux ont dégagé le sens - ou plutôt les sens - de ces images présentes sur internet. Parmi ces acteurs, il faut d'emblée distinguer entre: (1) l'Église où s'est déroulée la cérémonie, qui a tourné cette vidéo et l'a postée sur son site internet ; (2) Irregular Times, qui s'est saisi de cette séquence pour alerter le public; (3) et les médias généralistes, qui ont relayé les images, sans pour autant reprendre l'alerte du blog indépendant. Chacun de ces acteurs recourt à un usage différencié de la même vidéo en ligne pour s'adresser à un public différent. S'il est aisé de faire circuler un contenu médiatique sur la Toile, quelques clics suffisant à copier un fichier pour le diffuser plus loin, les pratiques interprétatives permettant la compréhension de ce contenu ne voyagent pas si facilement, en particulier lorsqu'il s'agit de franchir l'écart séparant des mondes culturels. Le travail d'interprétation intervient à ce point ; il vise à dégager le sens de ces images pour un public profane.

Malgré leur caractère exotique, les gestes accomplis durant cette cérémonie relèvent en grande partie d'une spiritualité ordinaire propre au pentecôtisme. Ce protestantisme accorde en effet une importance particulière à l'expérience extatique du Saint-Esprit et à la pratique tangible des dons spirituels: " la prophétie, la guérison [miraculeuse], l'exorcisme, le parler en langues et les révélations par le moyen de rêves ou de visions » (Anderson, 2013, p.8). «Imposer les mains » est une façon usuelle d'appeler la bienveillance divine, la «bénédiction", sur une personne. Cet attouchement peut

\footnotetext{
1 La vidéo «Sarah Palin Gets Protection From Witches » apparaît sur le compte « nyprogressive ", <https://www.youtube.com/watch?v=jl4Hlc-yfgM>, dernière consultation le 29 juin 2014.

${ }^{2}$ Cet article approfondit des analyses esquissées succinctement dans P. Gonzalez (2014, chap. 9).
} 
s'accompagner d'une injonction tentant de «lier» les puissances démoniaques, afin de « délivrer » le fidèle, raison pour laquelle les pentecôtistes préfèrent le terme « délivrance » à celui d'« exorcisme » (Gonzalez, 2014, p.51). Cependant, le caractère ordinaire de tels gestes qui, au départ, visent le "salut » d'un individu, interroge lorsqu'ils portent sur une personnalité : l'exorcisme cherche alors à atteindre la sphère dans laquelle elle exerce un rôle public. II apparaîtra bientôt qu'un tel déplacement, figuré par la vidéo de Palin, documente une transformation majeure du pentecôtisme.

Internet fut le lieu central pour la mise en visibilité de cette vidéo, sa diffusion et les interprétations qu'elle généra. En pensant la traduction culturelle, voire politique, qu'implique cette circulation rendue possible par le dispositif technique, je restituerai sa dimension sociale à cette production de l'information. Car diverses scènes de visibilité, liées à des mondes plus ou moins périphériques au sein de la société, prennent part à ce processus: les médias généralistes bénéficient d'une centralité dans l'espace public étasunien dont ne jouit pas la page internet de l'Église où furent postées les images. De même, cette circulation dépasse le cadre d'un espace public national, la nouvelle étant reprise par les médias étrangers - une reprise qui redouble le problème de la traduction culturelle de ce qui est donné à voir. L'entrée par internet permet donc d'observer la division sociale de la production et de la circulation de l'information, ainsi que de décrire le travail visant à générer des interprétations stabilisées d'interrogations relatives à la vie publique: ici, quelle signification politique accorder à « l'exorcisme » de Palin ?

Pour élucider cette question, je mènerai une « enquête à propos d'enquêtes » (Boltanski, 2012) visant à montrer comment les acteurs sociaux ont ressaisi la portée politique de la «bénédiction » de Palin. Après une brève exposition des éléments théoriques ayant guidé mon investigation, j'aborderai l'historique de la controverse, et m'arrêterai sur trois scènes de visibilité où ont été montrées ces images : un média généraliste de gauche, Countdown; Irregular Times; et l'Église charismatique qui a produit ces vidéos. Ces analyses me conduiront à évoquer comment une lecture politique se stabilise dans les principaux médias en 2011.

\section{Qu'on montre toujours quelque chose à quelqu'un}

Les notions qui ont guidé mon investigation se sont esquissées au fur et à mesure que je tentais de décrire comment s'y prenaient divers acteurs sociaux (responsables 
ecclésiaux, activistes, journalistes, etc.) pour dégager le sens public de certaines images de Palin prises dans un contexte religieux. L'analyse du corpus médiatique montrera combien les concepts théoriques que je m'apprête à aborder se rapportent aux gestes qu'effectuent les acteurs autour de cette controverse.

La première de ces notions relève de ce que j'appellerai des degrés d'exposition. Ce terme permet de penser différents niveaux de disponibilité pour un même matériau médiatique. Évoquant les contenus sur internet, Dominique Cardon (2010, pp.35-52) distingue entre ce qui est accessible, visible, ou encore public. L'accessibilité décrit des contenus présents sur le web, sans pour autant présager de leur degré de visibilité ; aucune barrière de sécurité n'entrave leur consultation. Certains contenus sont pourtant peu visibles : pour les trouver, il faut les chercher sciemment et, lorsqu'ils apparaissent dans les résultats suggérés par un moteur de recherche, ils figurent dans les tréfonds de la liste. La visibilité se rapporte donc à la façon dont la disponibilité d'un contenu est rendue plus ou moins saillante, notamment par des dispositifs techniques permettant d'organiser des recherches, mais aussi par les liens dont ils font l'objet sur les pages d'autres sites.

De même, une différence existe entre ce qui est visible et ce qui est public. Si la visibilité est liée à une disponibilité technique offrant une exposition variable à un contenu, ce même contenu acquiert un caractère public à partir du moment où il fait l'objet d'une attention particulière de la part d'un public, c'est-à-dire d'une communauté d'action et d'intérêt. Cette communauté se fait politique dès lors « qu'elle se constitue autour de l'observation et de l'exploration, de l'appréciation et de la régulation de certaines conséquences pour le "vivreensemble", des actions conjointes des membres d'une collectivité et des situations qu'elles créent 》 (Quéré, 2003, p.125).

Adjectif, «public ${ }^{3}$ qualifie la qualité d'un contenu (son degré d'exposition); substantif, il désigne un ensemble d'acteurs. Ce passage d'une acception à l'autre permet de mieux cerner les gestes que mettent en œuvre les acteurs sociaux lors de la controverse générée par la vidéo de Palin se faisant «bénir ». De fait, cette dernière formulation doit être amendée, car elle donne à penser que les images auraient par elles-mêmes suscité l'interrogation, l'embarras ou la réprobation. En réalité, cette séquence a produit cet effet parce qu'elle a été donnée à voir en dehors d'un contexte ecclésial

\footnotetext{
${ }^{3}$ Ma définition du public s'inspire des travaux de L. Quéré $(1992 ; 2003)$ et de L. Kaufmann (2008).
} 
bien particulier. La vidéo a fait l'objet d'une monstration, qui " est toujours une démonstration de quelque chose » (Dayan, 2011, p.193), et implique donc une transformation d'un "réel » de départ.

Arrêtons-nous quelques instants sur cette monstration, avec sa visée démonstratrice et ses effets transformateurs. En premier lieu, la monstration est un geste, accompli par quelqu'un, " transformant le visible en un spectacle qui appelle l'attention"; ce geste "suscite l'attention [d'autrui] et la module » (Dayan, 2009, p.25). Montrer une image, c'est l'inclure dans un propos pour en faire un élément d'un discours. Dans cette compréhension, le discours est une façon d'agir dans le monde et, simultanément, une manière de représenter ce monde, de même que les acteurs et les événements qui y interviennent (Widmer, 2010). L'acte de monstration transforme ainsi le "réel » dont l'image constitue une représentation, une trace et, plus profondément, une attestation ${ }^{4}$ : inscrire l'image dans un discours, c'est inviter les destinataires à s'y rapporter selon une perspective particulière, à se situer par rapport à ce qui est donné à voir et à entendre. Toutefois, le déploiement de la controverse sur les séquences exhibant Palin fera apparaître que toutes les monstrations ne se valent pas: les acteurs sociaux (avant même que les sociologues ne s'en mêlent) peuvent soumettre ces images à l'enquête, afin d'évaluer, outre leur authenticité, la validité des chaînes causales et des contextes d'arrière-plan convoqués par les discours qui prétendent expliquer la signification de ce qui est montré ${ }^{5}$.

«Les signes [tels les images] ne valent que pour autant qu'ils produisent des conséquences pour des individus engagés dans une entreprise commune ", c'est-à-dire des « communautés d'action » (Arquembourg, 2010, p.185). Cette observation n'est pas anodine ; elle présuppose que ceux qui montrent ces images le font en tenant compte du savoir partagé par leurs destinataires, un savoir indexé sur un horizon historique, social et culturel (Scannell, 1994 ; Widmer, 1999). La monstration anticipe donc certaines attentes, le fait qu'une révélation puisse intéresser un public particulier qui, par

4 Daniel Dayan (2013 a) y voit un fait considérable subvertissant l'idée même d'information, car toute monstration médiatique crée un fait accompli : s'il est possible de réfuter la désinformation, l'absence d'images montrées rend impossible le débat autour de l'information, un « réel » n'étant pas donné à voir.

${ }^{5}$ Sur ce point, je m'écarte de la conception de la vérité factuelle proposée par Dayan (2013 b, pp.25-41), l'auteur traitant ces enjeux via la figure du témoin, à laquelle il identifie le journaliste. Pour ma part, ce n'est pas sur l'évidence du témoignage oculaire que je fais reposer la production des évidences factuelles (même si le témoignage en est un élément essentiel), mais bien sur la démarche d'enquête, telle que la théorise John Dewey ([1938] 1993). 
exemple, souhaitera remédier à la transgression de certaines normes (Adut, 2005). L'usage stratégique de la publicité dépend justement de l'anticipation de ces attentes: des « entrepreneurs de visibilité » (Dayan, 2013 a, pp.143-145) tablent sur la possibilité de susciter l'attention publique, voire l'indignation d'un collectif, et même des sanctions institutionnelles.

Par ailleurs, si les images montrées conservent une consistance propre tout au long de leur transport, elles vont résonner différemment selon qu'elles apparaissent à une congrégation religieuse, à un groupe d'activistes de gauche ou auprès du public plus large d'un média généraliste, car chacune de ces collectivités mobilise un savoir différent. Dès lors, des pans de sens qui présidaient à la production d'une vidéo peuvent disparaître au gré de sa circulation. C'est le cas lorsque la séquence a été réalisée pour une communauté dont les pratiques échappent à la plupart de ses contemporains, ou lorsque les images sont vues par des spectateurs situés dans un contexte historique, social ou culturel différent (Gonzalez, 2013). Forts de ces élucidations théoriques, revenons à la controverse suscitée par les images de Palin.

\section{Palin candidate : l'appel à la Droite chrétienne}

Le 29 août 2008, Sarah Palin devient la première femme à briguer la vice-présidence des États-Unis d'Amérique. Le présidentiable John McCain dévoile le nom de sa colistière à la veille de la convention nationale du Parti républicain. La candidate est une relative inconnue pour l'opinion publique, bien qu'elle gouverne l'Alaska depuis fin 2006. Le choix de cette politicienne inexpérimentée se révèle payant : il suscite le ralliement d'un allié de taille, la Droite chrétienne qui, jusqu'alors, s'était montrée suspicieuse à l'endroit de McCain. Les positions très conservatrices de Palin - un décalque de la Christian Coalition ${ }^{6}$ - ont fini de rassurer le vote évangélique, essentiel à la victoire électorale ${ }^{7}$ : apologie du libre marché capitaliste; soutien inconditionnel à la National Rifle

\footnotetext{
${ }^{6}$ La Christian Coalition of America est fondée en 1989 par le télé-évangéliste Pat Robertson suite à l'échec de ce dernier dans la course à l'investiture républicaine pour la présidentielle de 1988. Elle prend le relais de la Moral Majority lancée en 1979 par le pasteur Jerry Falwell, et qui contribua à propulser Ronald Reagan à la présidence des États-Unis. Voir l'agenda politique de cette coalition : <http://www.cc.org/our_agenda>, dernière consultation le 29 juin 2014.

${ }^{7}$ Depuis la fin des années 1970 , le Parti républicain a noué des rapports étroits avec l'électorat évangélique (Williams, 2010). Près d'un Étasunien sur trois est évangélique, cf. Gonzalez (2014, introduction).
} 
Association afin de conserver le droit de porter une arme à feu (malgré les tueries qui défrayent régulièrement la chronique) ; opposition à l'avortement, ainsi qu'au mariage entre partenaires de même sexe (Barnes \& Shear, 2008 ; Bumiller \& Cooper, 2008 ; Shear \& Ellperin, 2008).

Les médias tentent d'en savoir plus sur la candidate, apportant leur lot de révélations quotidiennes. Le 3 septembre 2008 , en pleine convention nationale républicaine, la chaîne $A B C$ news diffuse une courte séquence où l'on voit Palin s'adresser à des coreligionnaires. Elle s'exprime devant l'Assemblée de Dieu de Wasilla [Wasilla Assembly of God, dorénavant «WAOG»], une Eglise charismatique dans laquelle elle fut baptisée à l'âge de douze ans et qu'elle a fréquentée jusqu'en 2002, avant de rejoindre une autre Église évangélique (Sataline, 2008 ; Johnson \& Severson, 2008 ; Palin, 2009, p.22). La vidéo, tournée en juin 2008 , quelques semaines avant la nomination sur le ticket républicain, figure parmi les célébrations que l'Église poste sur son site internet à l'endroit de ses fidèles ${ }^{8}$.

Sur le site d'ABC news, la séquence est intitulée «Palin : [la présence des] USA en Iraq est le "plan de Dieu" ». Elle s'accompagne d'un bref descriptif : "La gouverneure Sarah Palin invoque une puissance supérieure [higher power] pour expliquer la guerre en Iraq ${ }^{9}$. De même, $A B C$ news incruste un commentaire sur l'image, visible tout au long de la séquence : « l'attrait qu'exerce Palin sur les conservateurs » [Palin's appeal to conservatives]. Quant à la gouverneure, debout, à côté de la chaire, elle énonce micro en main : «Priez pour nos militaires, hommes et femmes, qui s'efforcent de faire ce qui est juste aussi pour ce pays, que nos leaders nationaux sont en train d'envoyer pour une tâche qui vient de Dieu ${ }^{10}$. Au terme de la séquence, un journaliste de la chaîne d'information présent à la convention républicaine commente ces propos : «Elle a dit que

8 Généralement, les vidéos de la WAOG sont accessibles ici : " Audio Vidéo ", $<$ http://www.wasillafirst.org/audio-video/>, dernière consultation le 29 juin 2014. En raison des controverses suscitées, les séquences montrant Palin ont rapidement été retirées du site Internet de la WAOG.

9 «Palin: U.S. in Iraq Is "God's Plan" », $A B C$ news, 03.09.2008, $<$ http://abcnews.go.com/Politics/video?id=5717948>, dernière consultation le 29 juin 2014.

${ }^{10}$ La formulation de Sarah Palin est ambigüe et sujette à deux interprétations, en particulier lorsqu'on restitue la phrase suivante de son discours : "Nous devons être sûrs que nous prions pour cela, qu'il y a un plan et que c'est le plan de Dieu » (Clifford, 2008a). La première interprétation serait que la guerre en Iraq est « une tâche [qui vient] de Dieu "; d'après l'autre lecture, il faudrait prier pour s'assurer que cette tâche vient vraiment de Dieu. Dans son autobiographie, Sarah Palin (2009, p.238) affirme que son intention correspondait à la seconde lecture. 
c'est le plan de Dieu que nous soyons allés en Iraq. Les évangéliques adorent ça. Je ne suis pas certain que le reste du pays soit aussi enchanté. »

Intrigués par l'arrière-plan religieux de la candidate, les journalistes suivent cette piste, s'intéressant à une allusion énoncée dans la version longue de la même vidéo: Palin attribue son succès électoral à la prière d'un pasteur nigérian, orateur régulier à la WAOG, Thomas Muthee. Dans un article documenté, le quotidien londonien, The Times met en évidence la notoriété internationale de ce prédicateur au sein du monde charismatique, tout en le qualifiant de « chasseur de sorcières » (Strange, 2008). En effet, Muthee prétend développer une intense activité contre les « entités démoniaques » et se vante d'avoir fait bannir de sa ville, Kiambu, une "sorcière ». La femme en question, Mama Jane, se disait « voyante » et a dû fuir l'agglomération de peur d'être lapidée, suite à des incidents violents avec les foules, et même à une descente de policiers venus tuer un « démon », un python, chez elle. Dans cette affaire, les prédications de Muthee n'ont pas manqué d'attiser les craintes en jouant sur le registre de la menace surnaturelle.

Le Times puise une part de ses informations dans une série de documentaires vidéo réalisée par une organisation évangélique nord-américaine, le Sentinel Group, et largement diffusée dans le monde charismatique. La série s'intitule Transformations et montre l'influence de la prière sur les villes de différents continents, l'ordre social étant "gagné à Christ ${ }^{11}$. Dans ce documentaire, Muthee affirme que la fuite de Mama Jane aurait constitué une victoire spirituelle sur les "forces démoniaques» qui tenaient la ville. Depuis lors, les accidents de voiture - causés par les «sorts» de la « sorcière »- auraient cessé.

Bientôt, les journalistes tombent sur une seconde vidéo de Palin, tournée elle aussi à la WAOG, mais autrement plus dérangeante. On y voit la politicienne trois ans auparavant, en 2005, au moment de briguer le mandat de gouverneur de l'Alaska. Le pasteur Muthee lui impose les mains pour la

\footnotetext{
11 Sur la page d'accueil de son site internet, cette organisation se présente ainsi : "Le Sentinel Group se consacre à "aider l'Église à prier en connaissance de cause pour l'évangélisation mondiale de la fin des temps, et à permettre aux collectivités [civiles] de découvrir un véritable réveil et une transformation sociétale". Le groupe a été formé en 1990 par George Otis Jr. Après avoir travaillé "à la pointe" des efforts d'évangélisation durant bien des années, [celui-ci] s'est inquiété de l'oppression spirituelle et de la persistance de cette oppression. Ses recherches sur ce problème l'on conduit à visiter plus de cinquante nations. " The Sentinel Group, <http://www.sentinelgroup.org>, dernière consultation le 29 juin 2014 (notre traduction).
} 
protéger de «toute forme de sorcellerie $»^{12}$. Le 23 septembre 2008, Irregular Times poste ces images sur Youtube (Clifford, 2008b). Elles sont aussitôt reprises par les médias du monde entier, dont $A B C$ News $^{13}$. Irregular Times n'est pas étranger à la publicisation de la première vidéo : la veille de la diffusion par $A B C$ news, le blog avait publié trois billets relatifs aux propos de Palin sur l'Iraq, dont l'un restituait la transcription intégrale de son discours à la WAOG (Clifford, 2008a).

En France, dès le 25 septembre 2008, le site Arrêts sur image parle d'une "vidéo virale " et titre "Palin protégée contre la sorcellerie " (Klein, 2008). La page présente les vidéos de l'allocution sur l'Iraq et de "l'exorcisme "; un renvoi à l'article du Times évoquant le pasteur Muthee est intercalé entre les deux séquences. Le même jour, L'Express propose un long papier renvoyant au Guardian (MacAskill, 2008), mais aussi à Irregular Times, ce dernier étant décrit comme un "site libéral » à l'origine du "buzz » (Simon, 2008). Le lendemain, Le Monde se contente d'une brève montrant la vidéo dans laquelle Palin reçoit « une bénédiction contre "la sorcellerie" de la part d'un prêtre [sic] kényan $"^{14}$. Pour Le Point, les deux séquences "dévoile[nt] une manifestation des profondes convictions religieuses de la candidate à la vice-présidence américaine " et susciteront probablement l'embarras du camp McCain (Larquier, 2008).

Ce bref historique fait apparaître des éléments d'arrière-plan importants pour comprendre le déroulement de la controverse sur la vidéo de la "bénédiction» disponible sur le web. L'identité "chrétienne » de Palin était une piste d'enquête majeure pour les journalistes, cette identité ayant fait l'objet d'une stratégie efficace de la part du camp McCain, qui avait ainsi su mobiliser la Droite chrétienne. Cet aspect religieux avait donc des incidences politiques : la première d'entre elles était électorale, mais elle était aussi liée aux valeurs « pro-vie » que la candidate comptait inscrire dans la loi.

Internet a dès lors constitué une ressource et un lieu importants pour conduire cette investigation. Les journalistes

12 Sarah Palin (2009) ne dit rien de Muthee ou de cette vidéo dans son autobiographie. Par contre, l'épisode fait l'objet d'un chapitre (Blumenthal, 2009) dans la biographie satyrique, bien qu'informée, que dirigent les éditeurs de The Nation (Kim \& Reed, 2009), un hebdomadaire de gauche.

13 «Palin Gets Anti-Witchcraft Blessing , ABC News, <http://abcnews.go.com/Politics/video?id=5885077>, dernière consultation le 29 juin 2014.

14 «Sarah Palin, bénie contre la sorcellerie », Le Monde, 26.09.2009, <http://www.lemonde.fr/elections-americaines/video/2008/09/26/sarah-palin-beniecontre-la-sorcellerie_1099739_829254.html>, dernière consultation le 29 juin 2014. 
ont appréhendé les sites web des Églises visitées par Palin comme des terrains privilégiés pour documenter la religiosité problématique de la candidate. Toutefois, ce média n'a pas seulement servi à collecter des pièces à verser au dossier ; il fut aussi une scène centrale de monstration: une part essentielle de l'activité des journalistes a consisté à attirer l'attention publique - interpellant les citoyens - sur des documents déjà présents sur internet, et donc accessibles, mais qui, jusqu'alors, ne jouissaient pas d'une telle visibilité en dehors des cercles évangéliques.

Pour autant, les convictions de Palin n'étaient que l'une des pistes suivies par les journalistes: les médias se sont aussi intéressés à son passé de maire de Wasilla (1996-2002), ou aux mesures économiques et écologiques qu'elle a mises en $œ u v r e$ en Alaska. Ainsi, dans les premières semaines de septembre, outre la question religieuse (Johnson \& Severson, 2008), le New York Times s'est penché sur la politique énergétique de Palin (Johnson, 2008) et sur le népotisme dont était entachée sa gouvernance (Becker, Goodman \& Powell, 2008).

Si la composante religieuse constituait un axe pour ressaisir la personnalité et la politique de Palin, l'analyse révélera que les médias généralistes n'étaient pas en mesure d'appréhender la signification de la vidéo de «l'exorcisme ». Les journalistes ont eu beau thématiser l'incongruité d'une politicienne se faisant «protéger de la sorcellerie », ils n'étaient pas équipés pour lire les images qu'ils avaient sous les yeux: il leur manquait une explication du savoir partagé propre à cette communauté religieuse, la WAOG, et aux réseaux charismatiques internationaux dans lesquelles elle s'insère.

Voyons à présent comment ces images ont été perçues en ces différents lieux. Notre investigation ira dans le sens inverse de l'exposition médiatique, de Countdown à la WAOG, en passant par Irregular Times.

\subsection{Countdown : la « bigote » et son « problème de pasteur »}

Le 19 septembre 2008, au lendemain de la parution de l'article du Times, l'émission d'actualité politique Countdown, sur la chaîne $M S N B C$, consacre une séquence aux rapports entre Palin et Muthee. Le présentateur vedette, Keith Olbermann, est connu pour ses analyses au vitriol du Parti républicain, au point que son émission est diffusée à la même heure que celle de son concurrent conservateur, Bill O'Reilly, sur Fox News. 
Olbermann se fonde sur les informations rapportées par le quotidien londonien. II propose un double cadrage pour lire la référence de Palin à Muthee dans son discours à la WAOG. Le premier cadrage se réfère à l'histoire et introduit le sujet, avant de montrer la vidéo de la gouverneure créditant le prédicateur de son succès électoral.

Lorsque, il y a près de 60 ans, le Sénateur Joe McCarthy et les républicains ont conduit une chasse aux sorcières métaphorique, [cette chasse] avait peut-être une qualité : jamais ils n'ont appelé effectivement qui que ce soit une "sorcière ". [...] il n'en va pas de même pour l'évangéliste étroitement associé à la candidate républicaine à la viceprésidence, au point que celle-ci lui attribue une part du mérite dans le fait d'être devenue gouverneure de l'Alaska.

L'incident avec Palin est comparé à l'un des pires moments de l'histoire du Parti républicain, la "chasse aux sorcières " que McCarthy mena, durant les années 1950, contre ses compatriotes suspectés d'activités communistes. Palin est toutefois présentée sous un jour moins favorable que le sénateur : l'entreprise à laquelle elle serait associée contre la sorcellerie n'a rien de métaphorique ; il s'agirait de défaire des "sorcières " bien réelles, dotées de pouvoirs maléfiques, le mot étant à entendre en son sens littéral. Les croyances de la gouverneure semblent dès lors étranges, voire dérangées.

Simultanément, Olbermann propose un autre cadrage, lié à l'actualité : le «problème de pasteur ». Cette catégorie s'affiche à l'écran, sous la photo de Palin, tout au long de la séquence. C'est une référence à des controverses qui ont ponctué la campagne des deux présidentiables : Obama a dû se distancer de certains propos tenus par le révérend Jeremiah Wright, son pasteur, un promoteur d'une théologie de la libération afroaméricaine, tout comme McCain a été obligé de désavouer l'un de ses soutiens, le pasteur fondamentaliste John Hagee, en raison d'allégations anti-catholiques. Olbermann utilise ainsi le cas de Palin pour montrer le caractère anodin des propos de Wright, par comparaison avec ceux de Muthee, tout en pointant le tapage dont avait fait l'objet le pasteur d'Obama dans les médias conservateurs.

Ces deux cadrages sont constants dans le traitement des vidéos de Palin à la WAOG. Ce fil conducteur organise l'échange entre Olbermann et une journaliste basée à Anchorage, invitée à, expliquer combien les croyances et les pratiques de cette Église semblent bizarres aux Alaskiens. L'idée d'une " guerre spirituelle » qui viserait à déloger les " démons » des cités, afin de faire baisser les statistiques de la criminalité, apparaît des plus saugrenues. Lorsqu'Olbermann 
demande si Palin a été interrogée à ce propos, son interlocutrice répond:

Nous essayons de nous focaliser sur la politique, parce que la vie privée des gens est très importante pour chacun de nous. Mais, dans le cas présent, avec le recul, je crois que nous aurions dû lui poser plus de questions à propos de son Église -parce qu'elle a une définition vraiment floue de la ligne de séparation entre l'Église et l'État - quand il s'est agi de sa gouvernance et de son action politiques ici.

À la fin de l'entretien, la journaliste s'interroge : comment Palin a-t-elle pu créditer Muthee de son élection ? Serait-ce le signe annonciateur d'un nouvel obscurantisme ? « [V]a-t-on se mettre à jeter des gens dans des puits pour voir s'ils flottent ", c'est-à-dire procéder à une ordalie ? Olbermann déclare alors : "C'est la réponse que j'attendais ".

Le 24 septembre 2008, Countdown consacre une seconde séquence aux rapports entre Palin et le pasteur Muthee. Jusque-là, la connexion avec la sorcellerie était indirecte : la gouverneure avait crédité un pasteur, décrit comme un "chasseur de sorcières " par les médias, de son succès électoral. Avec la vidéo mise en ligne par Irregular Times, dont Olbermann montre un court extrait, le lien devient direct: Muthee impose les mains à Palin et prie contre la "sorcellerie ». À nouveau, au moment d'introduire le sujet, le présentateur invoque le caractère littéral de cette " chasse aux sorcières » et les effets réels de cette traque :

Et puis il y a ce pasteur évangélique si étroitement lié à la gouverneure au point qu'elle lui attribue une part du mérite dans le fait d'être devenue gouverneure de l'Alaska. Le pasteur Thomas Muthee, vous vous souvenez, a fondé son Église en 1989, après être - littéralement - allé à la chasse aux sorcières à Kiyambu, au Kenya. II a accusé une femme du lieu d'être responsable des accidents de voiture, la taxant de "sorcière ", et la menaçant physiquement, avant de la chasser de la ville. Pas de métaphores, juste une chasse aux sorcières.

À présent, une vidéo a refait surface. On y voit le pasteur Muthee en train de prier [en imposant les mains] sur la maire Sarah Palin, dans [...] l'Assemblée de Dieu de Wasilla, en mai 2005. Elle est alors maire sortante. La préoccupation du pasteur pour les sorcières semble encore très présente.

Olbermann invite un collègue du Chicago Tribune à s'exprimer sur la séquence. L'échange porte aussi sur le "problème de pasteur" d'Obama, qui ne serait rien en comparaison de celui de Palin. L'invité ne manque pas d'ironiser sur la possible élection de la candidate à la vice- 
présidence: pense-t-elle amener ses «chasseurs de sorcières » à Washington pour exorciser le Capitole?

Countdown se veut une émission progressiste. Pourtant, son traitement reflète bien le cadrage proposé par les médias généralistes: cette «chasse aux sorcières » est présentée comme incongrue, parfois appréhendée avec dérision, et imputée à la personnalité «bigote » de Palin (la catégorisation demeure implicite), voire aux croyances « extravagantes » des «pentecôtistes» (ou « charismatiques»). Si cette lecture psychologisante (ou culturelle) a pour effet de décrédibiliser la candidate, elle n'en constitue pas moins, en 2008, l'interprétation principale qui s'impose de ces images rendues visibles sur le web, et auxquelles les principaux médias donnent une large publicité.

Cette monstration s'accompagne cependant d'une contrepartie: l'incongruité de la situation pour des profanes arrête très tôt l'enquête des médias généralistes, ces images étant traitées comme une occurrence supplémentaire de l'incompétence et de l'obscurantisme de Palin. Cet arrêt de l'investigation empêche de creuser les rapports entre le geste de Muthee et l'élection de la politicienne au poste de gouverneur. Et si cet exorcisme était autre chose qu'une « chasse aux sorcières »?

\title{
2.2. Irregular Times : “C’est la théocratie, idiote »
}

Irregular Times est l'une des sources importantes de la controverse. Il fournit des documents aux principaux médias, et en profite pour se faire sa propre publicité. Pourtant, ce blog propose un cadrage différent de ces images, comme dans ce commentaire qui accompagne la mise en ligne de la vidéo sur Youtube $^{15}$ :

\begin{abstract}
Cette nouvelle vidéo, disponible seulement depuis aujourd'hui (23 septembre 2008), montre Sarah Palin en train d'accepter une protection surnaturelle contre la sorcellerie de [la part de] Thomas Muthee, qui a aussi promu la campagne de Palin au poste de gouverneur comme une manière d'infiltrer le gouvernement avec l'agenda de la Droite religieuse de l'Assemblée de Dieu. Un truc de fou !
\end{abstract}

La «protection surnaturelle » serait à ressaisir dans un mouvement « d'infiltration » du gouvernement étasunien par la Droite chrétienne. L'une des difficultés de ce lexique tient au fait

\footnotetext{
15 «Sarah Palin Gets Protection From Witches », $<$ https://www.youtube.com/watch?v=j|4HIc-yfgM>, dernière consultation le 29 juin 2014.
} 
qu'il se rapporte à la révélation d'une cabale. La mise au jour d'une conjuration présuppose une enquête documentée et l'accès au matériau se révèle compliqué. Les lanceurs d'alerte risquent d'être perçus comme des paranoïaques adeptes de la «théorie du complot » (Boltanski, 2012). Pour lancer de telles accusations, un certain équipement s'avère nécessaire. Cet équipement est factuel, mais aussi institutionnel et énonciatif : une position au sein du champ médiatique permet d'asseoir le crédit des analyses énoncées, ce qui est loin d'être le cas pour Irregular Times. Mais, avant d'aborder la position de ce média, revenons sur le cadrage qu'il propose.

En septembre 2008, Irregular Times publie plus de cinquante billets sur Palin. Le blog épingle les travers (fondamentalistes) de la candidate, tels son soutien à l'enseignement du créationnisme ou son déni du réchauffement climatique. Son rédacteur principal, Jim Clifford, fouille la page consacrée aux sermons sur le site de la WAOG et débusque du matériel vidéo. II en profite pour mettre en ligne les séquences, comme la « bénédiction », et les accompagner de transcriptions.

Le billet paraît le même jour que la vidéo et s'intitule : "Transcription de Thomas Muthee qui apporte son soutien à Palin avec le plan d'"envahir" et d'"infiltrer" le gouvernement ». (Clifford, 2008c). À la différence de la notice sur Youtube, les termes renvoyant à l'infiltration sont mis entre guillemets pour signaler qu'il s'agit d'un discours rapporté. On peut ainsi lire les explications de Muthee aux fidèles à l'égard du geste qu'il s'apprête à accomplir sur Palin. Ces propos figurent dans la version longue de la vidéo, qui dure près de dix minutes.

Au terme de son billet, Clifford commente le discours de Muthee et prend position pour les citoyens étasuniens. Le journaliste souligne que Palin n'a pas été gênée par l'introduction du prédicateur. Or la candidate pourrait devenir présidente des États-Unis, si McCain venait à être élu et décédait pendant son mandat. Clifford énonce alors la « question que les électeurs ont le droit de poser : Sarah Palin marche-t-elle dans le plan de Thomas Muthee consistant à l'utiliser elle-même pour "envahir", "infiltrer" et "influencer" le gouvernement américain pour le compte du christianisme évangélique? »

Trois jours après cette publication, Clifford poste sur Irregular Times un nouveau billet fustigeant la couverture des médias généralistes: "C'est la théocratie, idiote: Fargen passe à côté sur Sarah Palin et Thomas Muthee » (2008 d). Clifford critique sévèrement Jessica Fargen, du quotidien conservateur Boston Herald, de même que le traitement de l'agence Associated Press et d'ABC News. Ces trois médias 
" passent à côté en se focalisant sur un mot, "sorcellerie" », car ils n'auraient tenu compte que des deux dernières minutes de la vidéo, sans s'intéresser aux explications de Muthee. Clifford corrige cette omission en fournissant le « compte-rendu politique substantiel » qu'il aurait fallu dégager de ces mêmes images:

Le sermon de Muthee (dont le thème est "Pourquoi Sarah Palin ? ") avance à plusieurs reprises que Sarah Palin doit être utilisée par le christianisme fondamentaliste comme un instrument pour « envahir » et « infiltrer » le gouvernement, et lancer des politiques qui placent le Dieu chrétien à la tête des écoles publiques, [...] des gouvernements des États, et [...] du gouvernement fédéral - à l'exclusion des membres des autres religions, tels les musulmans, les bouddhistes et les wiccans [néo-païens].

[...] C'est un programme politique. Thomas Muthee a délivré un sermon électoral en faveur de Sarah Palin (violant la loi fédérale d'exemption d'impôt [pour les Églises, tant qu'elles ne soutiennent pas un candidat]) qui proposait la complète démolition de la séparation entre Église et État, ainsi que l'installation d'une théocratie chrétienne en Amérique.

Clifford enfonce le clou en relevant que la teneur théocratique du propos n'a pas empêché Palin de venir recevoir sa « bénédiction » des mains du pasteur.

Malgré quelques amalgames, Clifford n'étant pas un spécialiste du religieux, l'analyse tient la route. On verra bientôt que l'emprise sur les institutions de la société est thématisée par certains courants de l'évangélisme. Toutefois, les principaux médias reprennent les images dénichées par Irregular Times sans accorder le même crédit à ses analyses. Parmi les raisons qui expliquent cette omission, on peut évoquer la position ambigüe de ce blog: les accusations de collusion sont lourdes et, malgré l'important travail mis en œuvre pour produire des faits, l'orientation progressiste d'Irregular Times et ses tirades virulentes contre la candidate républicaine conduisent les journalistes à répercuter ces informations avec circonspection. D'autant plus que les journalistes généralistes ou politiques connaissent mal le milieu religieux dans lequel se tient cette cérémonie. II faudra attendre la venue d'autres acteurs, perçus comme des spécialistes des agissements de la Droite chrétienne, pour que la lecture en termes de «menace théocratique » se stabilise et soit plus largement reprise. 
Irregular Times a donc accru la visibilité de la vidéo. II a procédé à une prémonstration (Dayan, 2013, p.143), dont le propre était d'escompter que ces images seraient relayées par des médias généralistes. Internet fut le lieu de cette visibilisation, qui connut un succès relatif : la séquence devint "virale », mais voyagea sans que le blog indépendant soit en mesure d'imposer son interprétation politique des images.

\subsection{Muthee à la WAOG : la « domination » pour horizon}

Comme une majorité d'Églises évangéliques, la WAOG dispose d'un site web sur lequel elle poste ses célébrations. Cependant, ses responsables se montrent rapidement embarrassés par la publicité - de nature critique - qu'attirent les vidéos de Palin dans leur chapelle. Ils coupent aussitôt l'accès à ces ressources et surveillent de près les personnes fréquentant leurs cultes, afin de débusquer d'éventuels journalistes qui se seraient glissés parmi les fidèles (Blumenthal, 2009). Ce n'est pas tant le contenu des messages de Muthee qui gêne les responsables ecclésiaux, mais le fait que des tiers, qui ne sont pas évangéliques, puissent s'émouvoir de ce qui est dit. En effet, l'explication prononcée par Muthee avant d'imposer les mains à Palin est susceptible d'une double écoute, selon que le destinataire soit un évangélique qui s'identifie au projet décrit par le prédicateur, ou qu'il s'agisse d'un public profane qui se sent ciblé par les velléités hégémoniques que porte ce discours.

Sa «bénédiction », Muthee lui donne le sens d'une "prise d'autorité » sur le gouvernement et la société, dans la perspective de hâter l'établissement du "Royaume de Dieu » sur Terre: «Lorsque nous parlons de la transformation d'une communauté [politique], nous parlons du fait que Dieu envahit sept domaines dans notre société. [...] C'est là que nous voyons le Royaume de Dieu infiltrer, influencer sept domaines de notre société. » Le prédicateur énumère ces aires: la religion, l'économie, la politique, l'éducation, les médias (il en omet deux, les arts et la famille, mais compte à nouveau la politique). Dieu prendrait le contrôle de la société grâce à l'action de chrétiens « intègres » qui, en accédant à la tête de ces « domaines », mettraient un terme « au genre de corruption dont on entend parler dans notre société ». Le pasteur décrit alors le mode opératoire pour la politique :

Il y a des gens qui sont faits pour la politique [dans nos églises], parce que Dieu veut prendre la dimension politique dans nos sociétés. Et il faut prier pour ces personnes. Voilà pourquoi je suis si heureux de voir Sarah ici. Nous devons prier pour elle, la 
soutenir. Et lorsque viendra le jour de voter, nous ne devons pas nous contenter de prier, mais nous devons être là. Je vous le dis, parce c'est ce que je dis dans notre Église [au Nigéria]. Je leur dis que nous avons besoin de cela au Parlement. Làbas, il y a des députés, des gouverneurs : nous avons besoin de frères là-dedans.

La proposition de Muthee fait passer ses destinataires croyants du statut de fidèles à celui d'activistes ou d'électeurs ${ }^{16}$, la lutte électorale et l'arène politique étant réinscrites au sein d'un affrontement cosmologique que Dieu mènerait contre les puissances démoniaques pour le contrôle de la société. Le geste de "bénédiction contre la sorcellerie " participe de ce « combat spirituel ». II vise à « libérer la voie » de Palin de toute entrave du démon.

Ces conceptions paraîtront certainement exotiques à un public profane (et à bien des chrétiens). Pourtant, elles sont partagées au sein de l'évangélisme charismatique auquel appartient Muthee. Ces idées ont notamment été diffusées, dès 1999, par la série de documentaires Transformations, à laquelle le pasteur nigérian a pris part. La " lutte » contre Mama Jane, la voyante de Kiambu, participait d'une restauration surnaturelle du monde social, l'ordre de la cité étant désormais placé sous le contrôle exclusif du Dieu chrétien et de ses "prophètes", comme en témoigne le comportement des policiers de Kiambu se prêtant à la "chasse aux démons". Cette "guerre spirituelle » constituerait une extension de l'évangélisation : le "réveil », la conversion en masse des individus ne suffit plus ; il faut la "réforme ", la conformation des institutions de la ville ou de la nation à la «Loi de Dieu». En 2000, Charisma Magazine, le mensuel charismatique étasunien, consacrait un long article à Muthee, louant l'impact de son action et de celle de ses collègues jusque dans les plus hautes sphères de l'État nigérian (Gaines, 2000).

Du territoire, la " guerre spirituelle » s'est déplacée vers des «domaines » ou des "sphères » au sein de la société, comme cela apparaît dans le discours de Muthee. Ces idées ont largement été théorisées depuis les années 1980 par C. Peter Wagner, fondateur de la "Nouvelle Réforme apostolique » [New Apostolic Reformation], un réseau d' " apôtres " et de «prophètes » charismatiques internationaux auquel appartient Muthee. Wagner synthétise ces conceptions dans un ouvrage intitulé Domination! Comment l'action du Royaume [de Dieu] peut changer le monde (2008). L'auteur reprend le plan de

${ }^{16}$ Pour des analyses similaires, voir J. Goldfarb (2006, chap. 6). 
bataille énoncé par le pasteur nigérian ; dans son livre, ce plan est appelé les « 7 Montagnes de la culture » (pp.147-148). Par contre, Wagner réfute toute velléité théocratique:

même si ma thèse est que le peuple de Dieu doit s'orienter de façon agressive vers la prise de domination [Dominion] sur la société et qu'il doit s'efforcer de placer les personnes avec les plus hauts standards bibliques aux charges gouvernementales, je ne souhaite pas donner à entendre que l'Église devrait gouverner. (p.15)

Le geste serait démocratique, car, dans tous les cas, «la majorité l'emporte » (ou « règne ») [majority rules] :

Pour être clair, s'il arrive que les chrétiens constituent la majorité dans une société démocratique, ils respecteront les droits des minorités. II ne faudra toutefois pas attendre d'eux dans une véritable démocratie - qu'ils soutiennent les désirs d'une minorité antichrétienne de façonner la société selon des valeurs non chrétiennes. [Ces chrétiens majoritaires] ne se sentiront non plus pas intimidés par ceux qui proclament que la religion ne devrait jamais être mélangée avec la politique. (p.17)

En réalité, la proposition de Wagner équivaut à un populisme théocratique $^{17}$, la morale religieuse de la majorité étant suffisante pour instaurer un ordre «chrétien » et le dicter au reste de la société. Cependant, ce populisme a conscience de la faiblesse de ses appuis populaires, en raison de l'érosion provoquée par la sécularisation des nations occidentales. Le plan des « 7 Montagnes » a ainsi été conçu pour assurer l'hégémonie culturelle et politique d'une minorité de chrétiens qui, parvenus aux postes à responsabilité au sein d'une société, imposeraient par le haut leurs catégories de perception, et donc leur domination, à la majorité des citoyens (Gonzalez, 2014, pp.287-293).

\section{La stabilisation de la lecture «dominioniste »}

En 2008, les médias généralistes n'étaient pas prêts à reprendre les analyses proposées par Irregular Times sur le web. Par contre, en 2011, ils parleront amplement du « dominionisme » de certains républicains, candidats (sérieux)

17 Je dois cette formule à J. Stavo-Debauge, doit on lira l'étude (2012) sur les faiblesses du libéralisme politique face aux attaques du fondamentalisme. 
à l'investiture de leur parti en vue de l'élection présidentielle. Ce «dominionisme » a trait aux relations troubles que ces politiciens entretiennent avec des tenants de la «théologie du Dominion ", membres de la Nouvelle Réforme apostolique de Wagner.

Cette adoption d'une lecture "dominioniste » est liée à des mutations profondes du Parti républicain, et en particulier à l'avènement du Tea Party, dont Sarah Palin est l'un des acteurs principaux. La forte présence de candidats issus de cette frange populiste, durant la course à l'investiture de 2011, conduit les journalistes politiques à s'interroger sur les liens ambigus qu'auraient noués ces républicains avec des courants religieux extrémistes. Ils ont alors recours à l'expertise des spécialistes de la Droite chrétienne. Ces spécialistes étaient déjà actifs en 2008, et postaient leurs analyses sur leurs sites internet: Religion Dispatches, Right Wing Watch, Talk to Action $^{18}$. Cependant, ils n'avaient pas capté l'attention des principaux médias lors de la controverse autour de la "bénédiction » de Palin, contrairement à Irregular Times qui avait su tirer profit de ses publications sur Youtube.

Les premiers jalons de cette collaboration entre sites internet de veille et médias généralistes ont été posés en 2008, alors que le geste de Muthee ne fait plus les gros titres. Le 24 octobre de cette année, le New York Times consacre un long article à la vidéo de Palin, dans lequel un spécialiste de Talk to Action, présenté comme un « chercheur », énonce les visées théocratiques du «combat spirituel 》(Goodstein, 2008). Bientôt, cette grille d'analyse se révèle pertinente pour couvrir l'actualité politique. En 2011, les médias généralistes ne proposent plus la lecture psychologisante (ou culturelle) de 2008, mais procèdent à une interprétation politique de la situation: ils traquent les signes indiquant des velléités hégémoniques, et scrutent la façon dont l'extrémisme religieux menace de radicaliser davantage encore le Parti républicain, déplaçant le centre de gravité de l'espace public étasunien vers les extrêmes.

Les éditorialistes conservateurs ne manquent pas de crier à la « paranoïa » et aux « théories du complot » lancées par les journalistes progressistes, tout comme Wagner, d'ailleurs (Gonzalez, 2014, ch. 9). Mais les enquêtes journalistiques, solidement étayées et corroborées par les recherches des spécialistes de la Droite chrétienne, produisent leurs effets,

$18 \quad$ Voir respectivement les sites: <http://religiondispatches.org> ; consultations le 29 juin 2014). 
révélant le populisme théocratique qui anime le Tea Party et mine le camp républicain.

\section{Ce que montre la monstration}

La vidéo de la «bénédiction» de Sarah Palin est un révélateur: sa circulation fait apparaître des disparités au sein du web et dessine une topographie différenciée dans la production, la circulation et la réception de l'information. Si notre analyse a porté sur un objet religieux, le phénomène qu'elle met en exergue concerne potentiellement tout contenu circulant sur internet. La monstration renvoie ainsi à la façon dont sont montrées ces images, mais aussi au lieu où elles sont données à voir : si cette façon leur confère un sens particulier, ce lieu leur assigne un degré de factualité. Ce degré varie considérablement selon que l'on se trouve sur un blog d'activiste ou sur un média généraliste, et il se répercute sur la signification de la vidéo. Ces variations se rapportent à des opérations auxquelles procèdent les «institutions de sens » (Certeau, 1985) que sont notamment les principaux médias. Ces institutions évaluent « ce qu'on dit », triant des énoncés qui circulent sur un mode proche de la rumeur. Au terme de ce tri, les énoncés éprouvés sont désormais pris en charge par un énonciateur institutionnel garantissant, pour une communauté donnée, "ce que nous savons". Et ici, quand bien même il s'agirait d'une vidéo - des images prétendant à une forme d'évidence - le procédé demeure analogue.

Notre enquête sur la monstration a restitué ces opérations de factualisation et de mise en sens, exhibant au passage les variations dans la crédibilité conférée à certains contenus. À cet égard, on peut parler d'une topographie de la crédibilité qui introduit des discontinuités épistémiques et sociales au sein du dispositif médiatique continu que constitue internet. Cette crédibilité se révèle pourtant à double tranchant: en tant qu'institutions de sens, les médias généralistes peuvent se contenter de fonctionner en validant certaines informations, tirant parti de la déférence qui leur est accordée (Kaufmann, 2008), tout en s'épargnant le travail d'une investigation approfondie, ce qui les conduit à produire des explications stéréotypées. L'opposition entre l'interprétation proposée par le blog Irregular Times et celle relayée par des médias généralistes tient notamment au privilège épistémique dont se nantissent ces derniers, alors qu'ils n'ont pas procédé, dans ce cas, à une analyse fine des images et du contexte dans lequel elles ont été prises. À cet égard, la reprise par le New York Times des recherches au long cours menées par des spécialistes travaillant sur la Droite chrétienne a permis 
d'élucider la dimension politique de l'« exorcisme » de Sarah Palin. Ce faisant, l'institution du sens a renoué avec la procédure qui fonde et justifie sa crédibilité : l'enquête ${ }^{19}$.

\section{Références bibliographiques}

ANDERSON A., 2013, To the ends of the earth: Pentecostalism and the transformation of world Christianity, New York, Oxford University Press.

ARQUEMBOURG J., 2010, «Des images en action. Performativité et espace public ", Réseaux, n¹63, pp.163-187

BARNES R. \& SHEAR M. D., 2008, « McCain Picks Alaska Governor; Palin First Woman on GOP Ticket ", The Washington Post, 29.08.2008, $<$ http://www.washingtonpost.com/wpdyn/content/article/2008/08/29/AR2008082901112.html>, dernière consultation le 29 juin 2014.

BECKER J., GOODMAN P. S. \& POWELL M., 2008, «Once Elected, Palin Hired Friends and Lashed Foes ", The New York Times, 13.09.2008, <http://www.nytimes.com/2008/09/14/us/politics/14palin.html?p agewanted=all>, dernière consultation 29 juin 2014.

BLUMENTHAL M., 2009, «The Witch-Hunter Anoints Sarah Palin ", dans R. KIM \& B. REED (dir.), Going rouge. Sarah Palin: an American nightmare, Deerfield Beach, Health Communications, pp.199-201.

BOLTANSKI L., 2012, Énigmes et complots. Une enquête à propos d'enquêtes, Paris, Gallimard.

BUMILLER E. \& COOPER M., 2008, "Advisers Say Conservative Ire Pushed McCain Away From Picking Lieberman ", The New York Times, 31.08.2008, $<$ http://www.nytimes.com/2008/08/31/world/americas/31iht31reconstruct.15764650.html?_r=2pagewanted=all\&pagewante $\mathrm{d}=$ all>, dernière consultation le 29 juin 2014.

CARDON D., 2010, La démocratie Internet: promesses et limites, Paris, Le Seuil.

CERTEAU M. de, 1985. "Le croyable, ou l'institution du croire », Semiotica, vol.54, n ^1-2, pp.251-266.

${ }^{19}$ Sur les rapports entre l'enquête et la pratique du journalisme ou des sciences sociales, cf. W. Lippmann ([1920] 2010) et J. Dewey ([1938] 1993). 
CLIFFORD J., 2008 a, «Text Transcript of Sarah Palin's Speech of June 8, 2008 at Master's Commission of Wasilla, Alaska ", Irregular Times, 02.09.2008, $<$ http://irregulartimes.com/2008/09/02/text-transcript-of-sarahpalins-speech-of-june-8-2008-at-masters-commission-ofwasilla-alaska/>, dernière consultation le 29 juin 2014.

CLIFFORD J., 2008 b, «Sarah Palin Had Witch Hunter Pray Against Witchcraft in Alaska! », Irregular Times, 23.09.2008, $<$ http://irregulartimes.com/2008/09/23/sarah-palin-had-witchhunter-pray-against-witchcraft-in-alaska/>, dernière consultation le 29 juin 2014.

CLIFFORD J., $2008 \mathrm{c}$, «Transcript of Thomas Muthee's Sermon Endorsing Sarah Palin as plan to "Invade" and "Infiltrate" Government », Irregular Times, 23.09.2008, <http://irregulartimes.com/2008/09/23/transcript-of-thomas-

muthees-sermon-endorsing-sarah-palin-2/>, dernière consultation le 29 juin 2014.

CLIFFORD J., 2008 d, "It's the Theocracy, Stupid: Fargen Misses the Point on Sarah Palin and Thomas Muthee ", Irregular Times, 26.09.2008, $<$ http://irregulartimes.com/2008/09/26/fargen-misses-the-pointon-palin-and-muthee-theocracy/>, dernière consultation le 29 juin 2014.

DAYAN D., 2009, "Sharing and Showing: Television as Monstration ", Annals of the American Academy of Political and Social Science, vol.625, pp.19-31.

DAYAN D., 2011, “L'impossible réalité brute », L'Homme, n०198-199, pp.191-212.

DAYAN D., 2013a, «Conquering Visibility, Conferring Visibility: Visibility Seekers and Media Performance ». International Journal of Communication, vol.7, pp.137-153.

DAYAN D., 2013b. Appearing in Public: Media and the Politics of Showing, New York, New School for Social Research («The William Phillips Lecture Series »).

DEWEY J., [1938] 1993, Logique. La théorie de l'enquête, trad. trad. G. Deledalle, Paris, Presses universitaires de France.

LARQUIER S. de, 2008, "REGARDEZ - Quand Sarah Palin est bénie par un "chasseur de sorcières" ", Le Point (Paris), 26.09.2008, <http://www.lepoint.fr/actualites-monde/2008-0926/regardez-quand-sarah-palin-est-benie-par-un-chasseur-desorcieres/924/0/277509>, dernière consultation le 29 juin 2014.

GAINES A., 2000, « Praying Down God's Power », Charisma Magazine, 31.12.2000, <http://www.charismamag.com/site- 
archives/189-features/africa/219-praying-down-gods-power>, dernière consultation le 29 juin 2014.

GOLDFARB J. C., 2006, The Politics of Small Things. The Power of the Powerless in Dark Times, Chicago, The University of Chicago Press.

GONZALEZ P., 2013, « La controverse avant le film : quelle politique pour Good Night, and Good Luck? ", Bulletin VALS/ASLA, vol.98, pp.39-59.

GONZALEZ P., 2014, Que ton règne vienne. Des évangéliques tentés par le pouvoir absolu, Genève, Labor et Fides.

GOODSTEIN L., 2008, “ YouTube Videos Draw Attention to Palin's Faith », The New York Times, 24.10.2008, <http://www.nytimes.com/2008/10/25/us/politics/25faith.html?_r =3\&pagewanted=all\&>, dernière consultation le 29 juin 2014 .

JOHNSON K., 2008, «The Unusual Challenges Palin Faced in Alaska ", The New York Times, 03.09.2008, <http://www.nytimes.com/2008/09/04/us/politics/04alaska.html? th $=\&$ emc $=$ th\&pagewanted=all>, dernière consultation le 29 juin 2014.

JOHNSON K. \& SEVERSON K., 2008, «In Palin's Worship and Politics, A Call to Follow the Will of God », The New York Times, 05.09.2008, <http://www.nytimes.com/2008/09/06/us/politics/06church.html? pagewanted=all\&_r=0>, dernière consultation le 29 juin 2014 .

KAUFMANN L., 2008, «La société de déférence. Médias, médiations et communication », Réseaux, vol.148-149, pp.79116.

KIM R. \& REED B. (dir.), 2009, Going rouge. Sarah Palin: an American nightmare, Deerfield Beach, Health Communications.

KLEIN G., 2008, «Palin protégée contre la sorcellerie (vidéo virale) », Arrêts sur image, 25.09.2008, <http://www.arretsurimages.net/breves/2008-09-25/Palinprotegee-contre-la-sorcellerie-video-virale-id1907>, dernière consultation le 29 juin 2014.

LIPPMANN W., [1920] 2010. Liberty and the news. Mineola, Dover Publications.

MACASKILL E., 2008, « US election: Sarah Palin featured in church video denouncing witchcraft », The Guardian (Londres), 24.09.2008,

<http://www.theguardian.com/world/2008/sep/24/uselections20 08.sarahpalin>, dernière consultation le 29 juin 2014. 
PALIN S., 2009, Going Rogue. An American Life, New York, HarperCollins Publishers.

QUÉRÉ L., 1992, «L'espace public : de la théorie politique à la métathéorie sociologique », Quaderni, vol.18, pp.75-92.

QUÉRÉ L., 2003, "Le public comme forme et comme modalité d'expérience », dans D. CEFAÏ et D. PASQUIER (dir.), Le sens du public, Paris, Presses universitaires de France, pp.113-134.

SATALINE, S., 2008, "Palin's Faith is Seen In Church Upbringing », The Wall Street Journal (New York), 04.09.2008, <http://online.wsj.com/news/articles/SB122048406528596987>, dernière consultation le 29 juin 2014.

SCANNELL P., 1994, "L'intentionnalité communicationnelle dans les émissions de radio et de télévision ", Réseaux, $\mathrm{n}{ }^{\circ} 68$, pp.49-63.

SHEAR M. D. \& ELLPERIN J., 2008, «With Palin On the Ticket, Evangelicals Are Energized », The Washington Post, 01.09.2008, $<$ http://www.washingtonpost.com/wpdyn/content/article/2008/08/31/AR2008083102171.html>, dernière consultation le 29 juin 2014.

SIMON M., 2008, «Sarah Palin, protégée contre la sorcellerie ", L'Express (Paris), 25.09.2008, <http://www.lexpress.fr/actualite/monde/amerique/sarah-palinprotegee-contre-la-sorcellerie_575504.html>, dernière consultation le 29 juin 2014.

STAVO-DEBAUGE J., 2012, Le loup dans la bergerie. Le fondamentalisme chrétien à l'assaut de l'espace public, Genève, Labor et Fides.

STRANGE H., 2008, "Sarah Palin Linked Her Electoral Success to Prayer of Kenyan Witch Hunter », The Times of London (repris par Alternet), 17.09.2008, <http://www.alternet.org/story/99118/sarah_palin_linked_her_el ectoral_success_to_prayer_of_kenyan_witch_hunter>, dernière consultation le $2 \overline{9}$ juin 2014.

WAGNER C. P., 2008, Dominion! How Kingdom action can change the world, Grand Rapids, Chosen Books.

WIDMER J., 1999, « Notes à propos de l'analyse de discours comme sociologie. La mémoire collective d'un lectorat ", Recherches en communication, vol.12, pp.195-207.

WIDMER J. 2010. Discours et cognition sociale. Une approche sociologique, Paris, Archives contemporaines. 
WILLIAMS D. K., 2010, God's own party. The making of the Christian right, Oxford, Oxford University Press. 